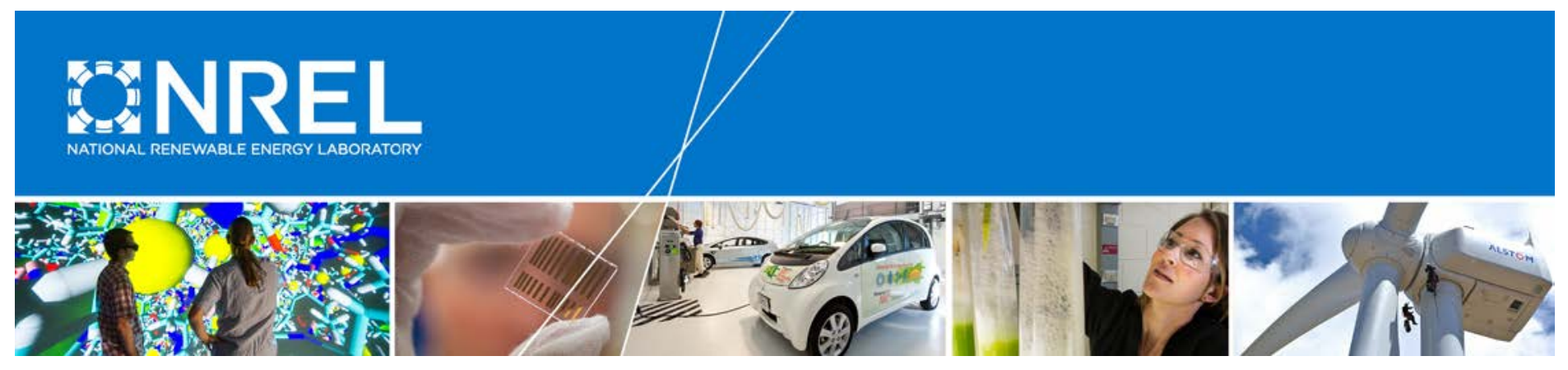

\title{
Validation Process Methods
}

John Lewis, Christine English, Josh Gesick, and Saikrishna Mukkamala National Renewable Energy Laboratory

NREL is a national laboratory of the U.S. Department of Energy Office of Energy Efficiency \& Renewable Energy Operated by the Alliance for Sustainable Energy, LLC

This report is available at no cost from the National Renewable Energy Laboratory (NREL) at www.nrel.gov/publications.

\section{Technical Report}

NREL/TP-6A20-70329

January 2018 


\section{Validation Process Methods}

John Lewis, Christine English, Josh Gesick, and Saikrishna Mukkamala National Renewable Energy Laboratory

Prepared under Task No. 6A20.10083.01.01.05

NREL is a national laboratory of the U.S. Department of Energy Office of Energy Efficiency \& Renewable Energy Operated by the Alliance for Sustainable Energy, LLC

This report is available at no cost from the National Renewable Energy Laboratory (NREL) at www.nrel.gov/publications.

National Renewable Energy Laboratory 15013 Denver West Parkway Golden, CO 80401

303-275-3000 • www.nrel.gov

\section{Technical Report}

NREL/TP-6A20-70329

January 2018

Contract No. DE-AC36-08GO28308 


\title{
NOTICE
}

This report was prepared as an account of work sponsored by an agency of the United States government. Neither the United States government nor any agency thereof, nor any of their employees, makes any warranty, express or implied, or assumes any legal liability or responsibility for the accuracy, completeness, or usefulness of any information, apparatus, product, or process disclosed, or represents that its use would not infringe privately owned rights. Reference herein to any specific commercial product, process, or service by trade name, trademark, manufacturer, or otherwise does not necessarily constitute or imply its endorsement, recommendation, or favoring by the United States government or any agency thereof. The views and opinions of authors expressed herein do not necessarily state or reflect those of the United States government or any agency thereof.

This report is available at no cost from the National Renewable Energy Laboratory (NREL) at www.nrel.gov/publications.

Available electronically at SciTech Connect http:/www.osti.gov/scitech

Available for a processing fee to U.S. Department of Energy and its contractors, in paper, from:

\author{
U.S. Department of Energy \\ Office of Scientific and Technical Information \\ P.O. Box 62 \\ Oak Ridge, TN 37831-0062 \\ OSTI http://www.osti.gov \\ Phone: 865.576.8401 \\ Fax: 865.576.5728 \\ Email: reports@osti.gov
}

Available for sale to the public, in paper, from:

\author{
U.S. Department of Commerce \\ National Technical Information Service \\ 5301 Shawnee Road \\ Alexandria, VA 22312 \\ NTIS http://www.ntis.gov \\ Phone: 800.553 .6847 or 703.605 .6000 \\ Fax: 703.605.6900 \\ Email: orders@ntis.gov
}




\section{Executive Summary}

This report documents the validation process that is applied to projects awarded through funding opportunity announcements (FOAs) within the U.S. Department of Energy Bioenergy Technologies Office (DOE-BETO). It describes the procedures used to protect and verify project data, as well as the systematic framework used to evaluate and track performance metrics throughout the life of a project. This document also describes the procedures used to validate the proposed process design, cost data, analysis methodologies, as well as supporting documentation provided by the recipients.

The three main objectives of this validation process are:

- Implement the established validation process framework to evaluate and track performance metrics throughout the project

- Work with all stakeholders (DOE-BETO, support contractors, project teams, subject matter experts, and independent engineers) to align quantifiable performance metrics with FOA and program objectives

- Provide independent, credible, and objective technical and economic analysis of project technologies to inform decisions.

This report addresses the following comment and recommendation from the 2015 BETO Peer Review Panel for the conversion program: "The Peer Review Panel was unanimous regarding the importance of the validation process, and considers it to be a strength of the program, and recommends that the process be continued, and the overall procedure accurately documented" (DOE 2016). The report serves as the accurate documentation of the validation process for DOEBETO. Other applications of this validation process overview report include:

1. Providing guidance for the development of validations plans for future FOAs

2. Ensuring consistency in the implementation of the validation process across the office

3. Providing guidance for DOE-BETO technology managers unfamiliar with the validation process. 


\section{Data Protection and Confidentiality Agreements}

Before accessing any project related data or performing any validation work, all members of the validation team enter into a written confidentiality agreement that covers any confidential and business sensitive information that they may be exposed to during the validation process. All communications between DOE-BETO, the validation team, and the project team personnel related to the validation adhere to the stringent conflict of interest and non-disclosure agreement conditions covering the work. An example of the confidentiality agreement documents signed by the validation team personnel is contained in Appendix A. The recipient is free to negotiate a separate confidentiality agreement with the entities (typically national laboratories) representing the members of the validation team. However, the confidentiality agreement documents included in Appendix A have been considered adequate by most recipients that have undergone validation, and the documents have been approved by legal staff in the DOE Golden Field Office. The decision regarding confidentiality agreements is ultimately the recipient's. Note, however, that the validation team does not undertake the validation effort until there is agreement on use of these or other confidentiality agreement documents. Should a separate confidentiality agreement document be desired, negotiation must be completed in a timely manner so as not to impact the validation and project schedule. 


\section{Table of Contents}

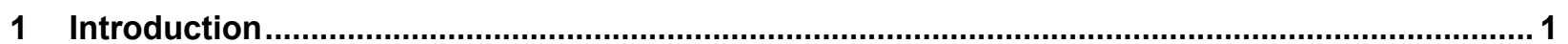

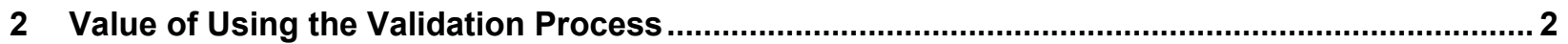

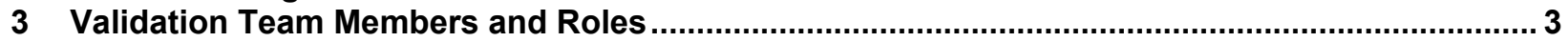

4 Overview of Validation Process

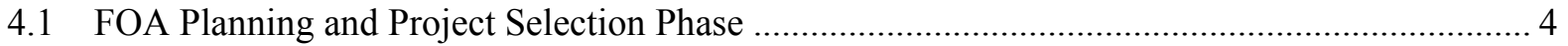

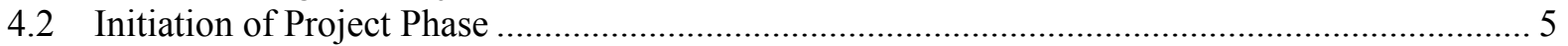

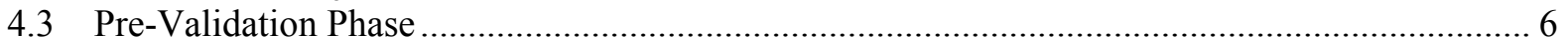

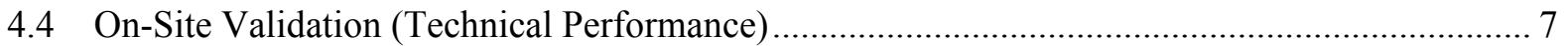

4.5 On-Site Validation (Process Design and Economics) ............................................................. 7

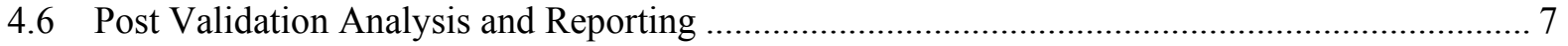

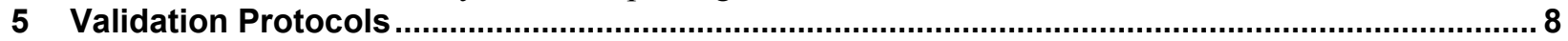

5.1 Protocol for Proposing Changes (Change Control Protocol) ............................................... 8

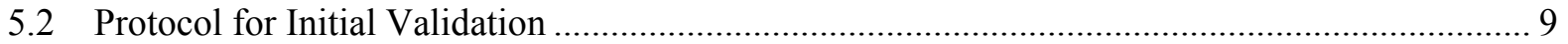

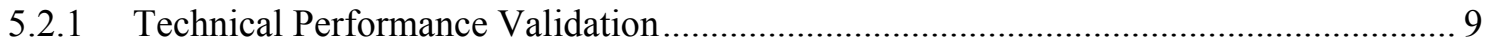

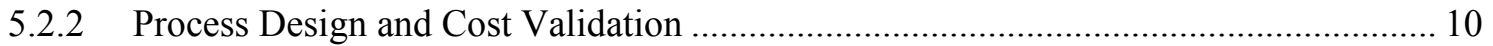

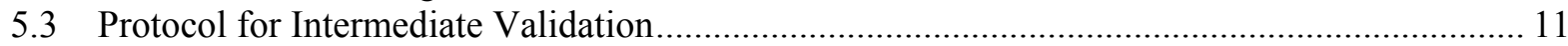

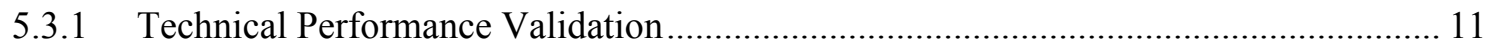

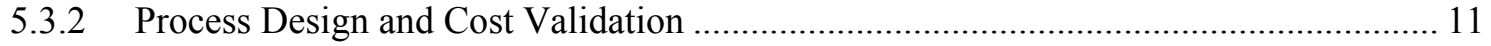

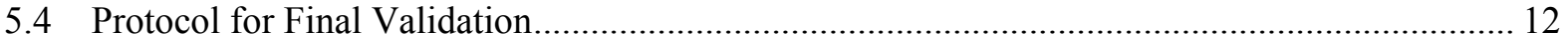

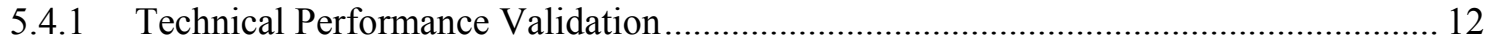

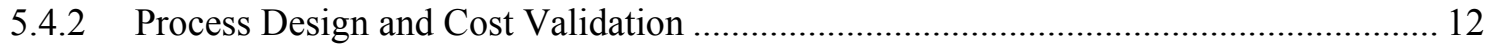

5.5 Protocol for Dealing with Unsuccessful Validations .......................................................... 12

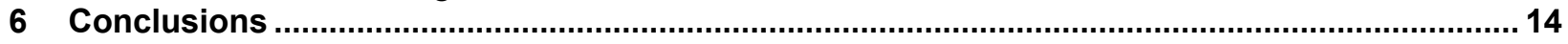

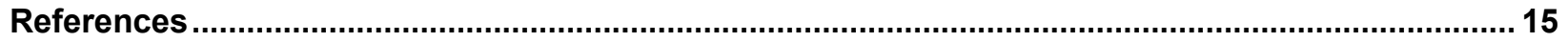

Appendix A: Examples of Confidentiality Agreement Documents ............................................. 16

Appendix B: Example of TechFin Tables Template ................................................................. 17

Appendix C: Example of a FOA, including Language Regarding the Validation Process ................ 18

Appendix D: Example of a FOA-Specific Validation Plan .............................................................. 19

Appendix E: Example of a Project-Specific On-Site Requirements Document................................ 20

\section{List of Figures}

Figure 1. Validation process summary as incorporated into DOE-BETO's FOA process ................. 2

Figure 2. Value of the project validation process for DOE-BETO and project teams .......................... 3

Figure 3. Overview of phased validation process ......................................................................... 5

Figure 4. Three elements occurring within each of the three validation phases: initial, intermediate,

and final 


\section{Introduction}

Validations are well-established project management and product acquisition tools within the discipline of systems engineering, and they are routinely applied in the defense and aerospace sectors (DoD 2017; DOE-BETO 2008; NASA 2007). The specific validation methodology documented in this report was adapted by the National Renewable Energy Laboratory's Systems Integration (NREL-SI) team from such traditional product development practices to track the technical and economic performance of chemical process technologies for DOE-BETO. However, the overarching validation process goal of applying a systematic approach to supplant risk over time remains consistent in this manifestation of the validation process as applied to developing chemical processes.

The major objectives of the validation process applied to U.S. Department of Energy Bioenergy Technologies Office (DOE-BETO) funding opportunity announcement (FOA) projects include:

- Clarify and establish a common interpretation of key project performance metrics with all stakeholders

- Provide continuity and consistency across the portfolio of projects to support portfolio analysis and decision making

- Establish, compile, analyze, and validate project-level technical and economic performance metrics

- Verify project performance and progress over the life of a project

- Compare project performance to the current state-of-technology benchmarks and projections on a variety of technical, economic, and environmental dimensions

- Compare different technologies and pathways across the DOE-BETO investment portfolio

- Monitor and track technical progress of individual projects and technology pathways

- Provide credible and objective data analysis to inform internal decision making and planning processes.

The above list represents a menu of options for implementing the validation process; different programs within DOE-BETO have chosen to tailor the validation process to their specific FOA and program needs, as the situation requires. Application of this validation process ranges from a complete implementation by the NREL-SI team for some conversion and algae program FOAs to sharing implementation with the independent engineers of a tailored validation process on some demonstration and market transformation program FOAs. The validation team works with all stakeholders, including DOE-BETO, NREL-SI, independent engineers, support contractors, and project teams to establish, compile, validate, analyze, and report results. The validation process flow, along with key outputs, is summarized below in Figure 1. The validation process is separated into three phases: (1) work with stakeholders to tailor and align key project performance metrics with project and program goals to ensure continuity and consistency across the portfolio, (2) collect, review, and validate key project performance data as well as identify the design basis and state of technology by evaluating the number of tests performed, feedstock used, scale and duration of tests used to establish basis data, and (3) complete analysis and generate reports and dashboards comparing individual projects across the overall portfolio. 


\begin{tabular}{|c|c|c|}
\hline $\begin{array}{l}\text { Work with } \\
\text { Stakeholders }\end{array}$ & $\begin{array}{c}\text { Collect and } \\
\text { Validate Data }\end{array}$ & $\begin{array}{c}\text { Analyze Data to } \\
\text { Inform } \\
\text { Decisions }\end{array}$ \\
\hline 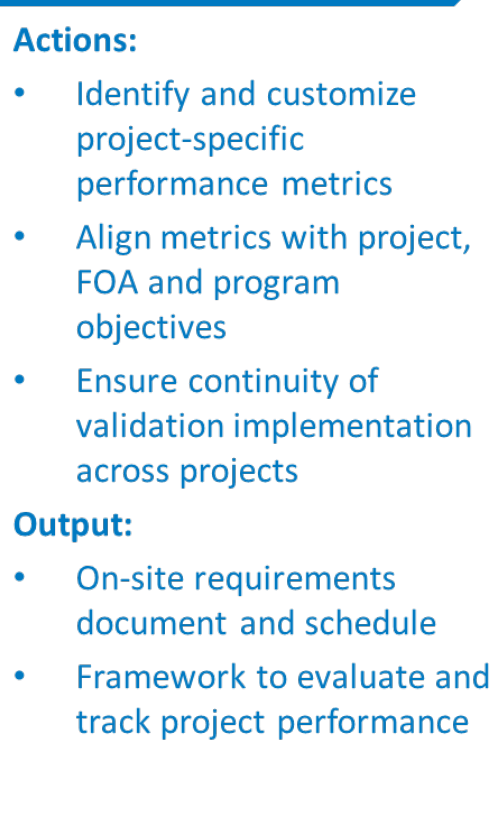 & $\begin{array}{l}\text { Actions: } \\
\text { - } \quad \text { Collect and review data } \\
\text { Observe experiments (if } \\
\text { required) } \\
\text { - } \quad \text { Verify benchmark data } \\
\text { basis and state of } \\
\text { technology, e.g., hours } \\
\text { of operation, number } \\
\text { of tests, scale, and } \\
\text { duration of tests } \\
\text { Output: } \\
\text { - Validated data, process } \\
\text { design, and process } \\
\text { economics } \\
\text { - Assessment of } \\
\text { sufficiency of project } \\
\text { facilities and resources }\end{array}$ & $\begin{array}{l}\text { Actions: } \\
\text { - Compare experimental } \\
\text { and operational } \\
\text { performance to project } \\
\text { and program targets } \\
\text { (gap analysis) } \\
\text { - } \quad \text { Evaluate economic } \\
\text { assumptions and bases } \\
\text { - Compile project-level } \\
\text { data for portfolio-level } \\
\text { analysis } \\
\text { - Generate dashboards } \\
\text { and reports } \\
\text { Output: } \\
\text { - Independent, credible, } \\
\text { and objective analysis } \\
\text { of project technologies } \\
\text { to inform decisions }\end{array}$ \\
\hline
\end{tabular}

Figure 1. Validation process summary as incorporated into DOE-BETO's FOA process

\section{Value of Using the Validation Process}

The validation process, as adapted for DOE-BETO, is a tool for discovering vital information regarding the technical and economic viability of a proposed process, and it benefits both DOEBETO and the project team. DOE-BETO gains in-depth technical and economic insight about the project that is not achieved during the application review process. The project teams benefit from independent third-party analysis of their technology and receive valuable input they may not have previously considered. A summary of benefits realized through this validation process over the five-plus years it has been applied to DOE-BETO FOA projects is shown in Figure 2 (next page).

Portfolio-level analyses have been performed using validation data gathered during select conversion and algae program FOAs. Portfolio-level analysis was performed using Biological and Chemical Upgrading for Advanced Biofuels and Products (DE-FOA-0001085) and MEGABIO (DE-FOA-0001433) validation data to compare state-of-technology and multi-year program plan goals to project metrics demonstrated during validations. Conversion project metrics compared to goals included conversion yields, minimum fuel selling prices, and carbon conversion efficiencies. For the algae program, validation data collected during the ABY (DEFOA-0000811) projects was evaluated to understand progress to date toward the FOA objectives. Specifically, algae validation data was analyzed to document progress toward meeting algal productivity, biomass conversion efficiency, as well as harvesting and dewatering performance goals. DOE-BETO staff members were briefed on the findings of both of these portfolio-level analyses during FY17. 


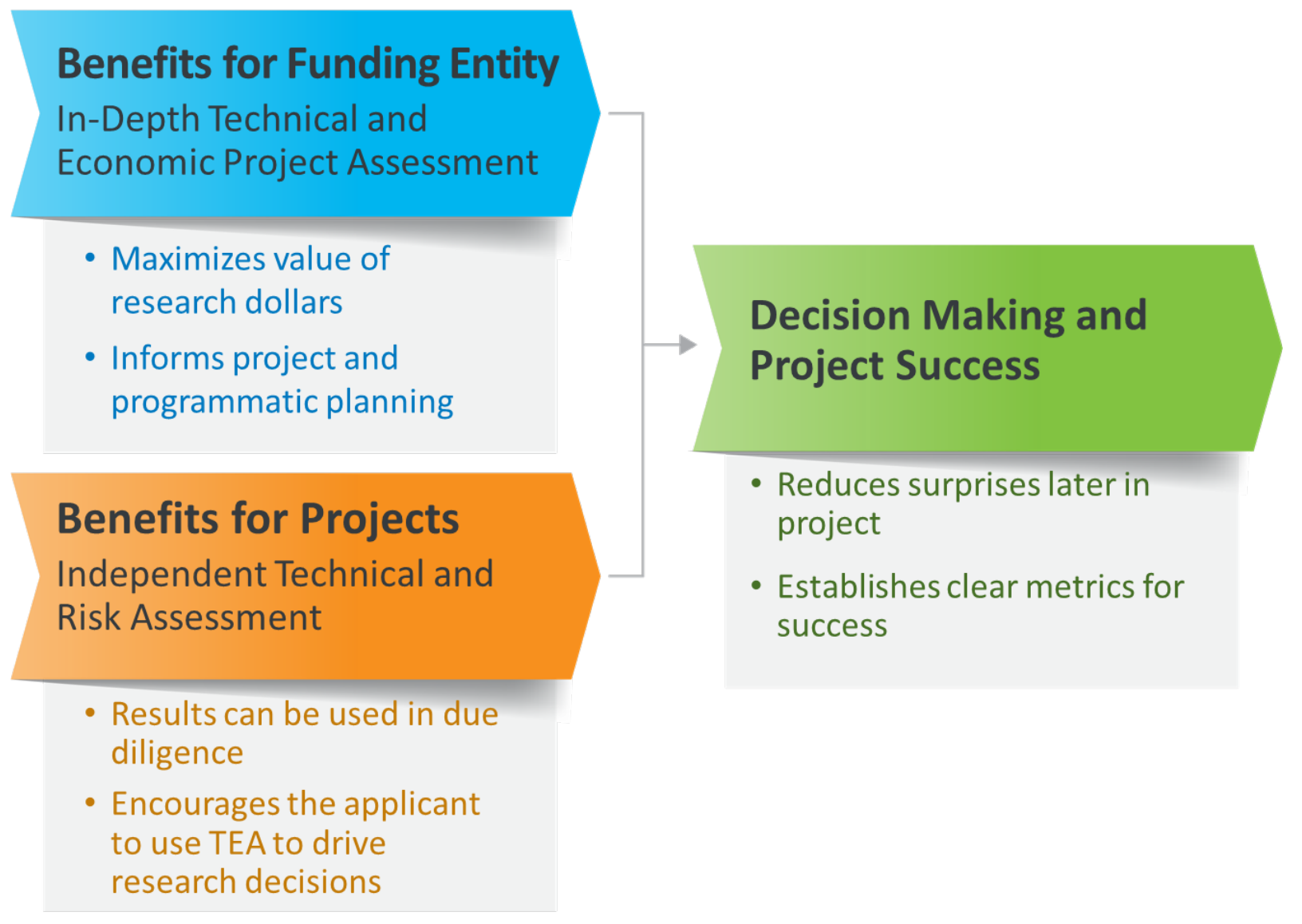

Figure 2. Value of the project validation process for DOE-BETO and project teams

\section{Validation Team Members and Roles}

The goal of the validation process is to provide independent, credible, and objective analysis (both technical and economic) that can be used to inform decisions. This objective is primarily achieved by (1) developing a framework to evaluate and track project performance and (2) informing stage-gate reviews and go/no-go decisions. The responsibilities of the validation team are typically shared by three people with the following roles and responsibilities:

- The validation lead is responsible for managing and coordinating the validation effort to provide continuity and consistency across all projects. The validation lead is responsible for working with all stakeholders to ensure key performance metrics are aligned with (1) DOEBETO expectations, (2) project objectives, (3) FOA objectives, and (4) program objectives. The role of validation lead is typically filled by a member of the NREL-SI team or team of independent engineers.

- The technical lead/subject matter expert is responsible for documenting and reporting on technical demonstrations and for providing technical advice and input on all aspects of the technical validation.

- The process engineer and techno-economics lead is responsible for reviewing the process design and economic calculations, as well as documenting and reporting techno-economic observations and results. 
The validation team is responsible for (1) implementing the DOE-approved validation plan, (2) working with the recipients to collect and analyze the relevant performance and techno-economic cost data, and (3) when appropriate, providing recommendations or advice to DOE-BETO regarding the interpretation of the analysis results. DOE-BETO is responsible for (1) considering the validation team's analysis and any recommendations and advice, (2) considering appropriate information from other sources, such as the project's quarterly reports, if available, and (3) making any decisions and taking appropriate actions based on the total gathered information that involve the administration of the financial assistance award management, such as funds availability and granting of continuations or extensions.

All communications and correspondence between the validation team and the project team include the principal investigator or principal investigators identified in the DOE-BETO contract (or their designee). The validation team and principal investigators verify contact information for all parties (e.g., phone, email, and mailing address) at the start of the validation process. In addition, the details of the validation process are reviewed with the principal investigator, including procedures for communicating changes or dealing with unsuccessful validation visits.

\section{Overview of Validation Process}

This section documents each phase of the validation process from FOA planning and project selection to post validation analysis and reporting.

\subsection{FOA Planning and Project Selection Phase}

The validation process is initiated during the planning phase of the FOA. Thus, the first activity in a validation occurs when the NREL-SI team works with the FOA manager to understand the scope of the FOA. The NREL-SI team then works with the FOA manager to create the template for capturing the technical and process economic data that applicants submit as part of their applications. Once this template is populated with data by the applicant, it becomes known as the technical and financial (TechFin) tables. Appendix B contains an example of an unpopulated TechFin template that was created for a FOA announced in 2016. It should be noted that development of the TechFin template is an iterative process whereby the NREL-SI team creates the tables and gathers edits to the tables suggested or requested by DOE-BETO and/or support contractors working on the FOA. The tables need to be adjusted for each FOA based on technology readiness level, types of technologies included in the project, and other criteria. During the FOA planning phase, the NREL-SI team works closely with the FOA manager to include language in the FOA document pertinent to the validation process and requirements. Appendix $C$ (pages 3-6, 8, 23, 33, 53, 74, 78-81) provides an example of how the validation process was integrated into a FOA published in 2016, and it includes language regarding the validation process.

The next step in the validation process occurs once the projects for a FOA have been selected and announced. At this point, the NREL-SI team develops a validation plan for performance assessment of all of the projects awarded under the specific FOA. The validation plan is distributed to the projects awarded under the FOA by the FOA manager. This validation plan document describes the procedures that are used to verify the data and protocols provided by the recipients in addressing the criteria in the FOA. It is important to note that this document is specific to the FOA. It is not, however, specific to each project. The goal of this document is to 
communicate expectations on DOE-BETO's behalf to all project recipients. An example of a validation plan produced for a FOA published in 2016 has been included as Appendix D.

\subsection{Initiation of Project Phase}

During the life of a project, there are three validation phases during which three on-site visits are made to the recipient's facility to validate reported technical performance and related technoeconomic projections. The objective of the first ("initial") on-site validation is to confirm the benchmark information provided in the application, which establishes the baseline against which future performance and cost improvements are evaluated. A second ("intermediate") on-site validation is conducted before a go/no-go or stage gate review of the project to assess progress relative to the intermediate performance and cost targets. A third ("final") on-site validation visit is scheduled if a successful go or stage gate result was achieved after the intermediate validation.

A summary of the phased validation process, along with an approximate timeline, is provided in Figure 3. As shown in the figure, the primary objective of each phase is to validate the performance progress, process design details, and associated process cost estimates. A secondary objective is to review the sufficiency of the project facilities, along with quality assurance and quality control procedures.

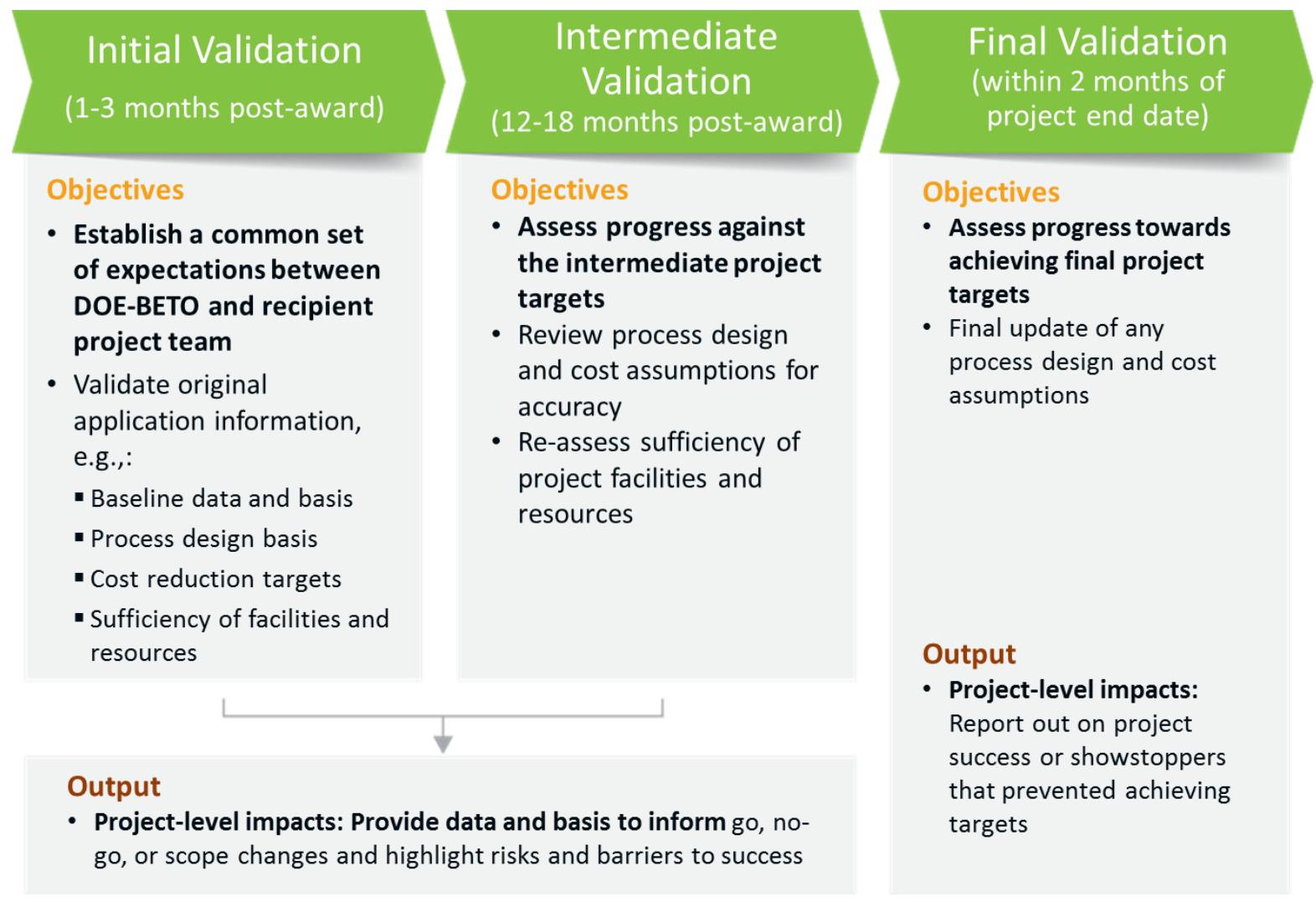

Figure 3. Overview of phased validation process

Within each of the three validation phases (initial, intermediate, and final), there are three elements as shown in Figure 4. Each validation has a pre-validation phase element, an on-site 
validation element, and a post-validation analysis/reporting element. The details of each of these elements are detailed below.

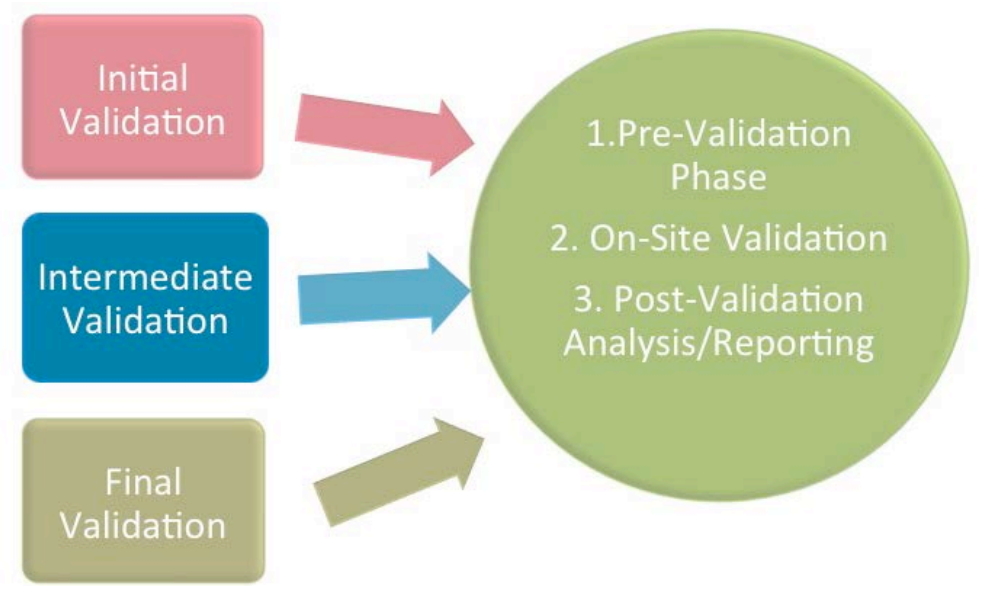

Figure 4. Three elements occurring within each of the three validation phases: initial, intermediate, and final

\subsection{Pre-Validation Phase}

Pre-validation work occurs before each on-site visit so that the scope of work for the validation visit can be defined. Elements of this pre-validation work include:

- Assemble the validation team by identifying and appointing appropriate personnel to fill the required roles

- Provide time for the validation team to review the project application materials, TechFin tables, and statement of project objectives document; these documents are essential for the validation team as it develop its project-specific validation documents and initiate iterations on metrics and key project performance metrics during the pre-validation phase.

- Conduct an overview of the validation process with the project team

- Work with all stakeholders to develop a project specific on-site requirements document; it should be noted that the project-specific on-site requirements document is different from the FOA specific validation plan. The on-site requirements document outlines what the validation team expects to see and discuss during the on-site visit. It sets expectations for specific experimental demonstrations, analytical demonstrations, techno-economic calculations, and facility tours, for example. A sample on-site requirements document is included as Appendix E to demonstrate the level of project specific detail included in this document.

- Set a schedule for the on-site validation visit with input from all stakeholders

- Develop an agenda for the on-site visit.

This pre-validation work is typically achieved via a series of conference calls between all stakeholders. The kickoff conference call initiates the validation process by introducing all stakeholders, explaining the validation process, and setting expectations for the validation visit. Participants in the pre-validation conference calls also review the validation plan and on-site requirements document, set the dates for the on-site validation visit, and work out the logistics 
of the validation visit. If needed, the validation team may request additional supporting documentation, revisions to the TechFin tables, or additional clarifications. Completing these items before the on-site validation visit has been shown to improve the focus and productivity of the on-site validation visit.

\subsection{On-Site Validation (Technical Performance)}

The objective of the on-site technical performance validation is to validate the technical application data by physically demonstrating the data included in the project application. The responsibilities of the project team include (1) demonstrating the process operations as described in the application and/or negotiated in the award, (2) reproducing the application data (or data agreed to during award negotiations) to within a coefficient of variation of $5 \%$, and (3) submitting a summary of experimental results once data analysis is complete. The responsibilities of the validation team include (1) making detailed observations of the demonstrated process operations to ensure replication of what was included in the original application or negotiated award, (2) making observations and recommendations for the project, and (3) noting any technical variances, errors, or other inconsistencies in the application data and demonstrated data.

\subsection{On-Site Validation (Process Design and Economics)}

The objective of the on-site process design and economics validation is to validate the process design and economic data included in the application. This is achieved by demonstrating the techno-economic calculations and models for the process and providing supporting literature references and data. The responsibilities of the project team include (1) demonstrating the techno-economic calculations and models described in the application and/or negotiated in the award, (2) replicating the application data (or data agreed to during award negotiations) to within a coefficient of variation of 5\%, and (3) submitting a summary of techno-economic results after data analysis is completed. The responsibilities of the validation team include (1) making detailed observations of the demonstrated techno-economic calculations and models to ensure replication of what was included in the original application or negotiated award, (2) making observations and recommendations for the project, and (3) noting any techno-economic variances, errors, or other inconsistencies in the application data and demonstrated data.

\subsection{Post Validation Analysis and Reporting}

The results of each phase of the validation (initial, intermediate, and final) for a project are reported to DOE-BETO via two methods:

1. A one-page synopsis intended to quickly inform negotiations for the next budget period - This one page document includes two bulleted lists that highlight key observations (including potential risk areas), and recommendations that DOE-BETO may consider in negotiating scope and budget in the next budget period. The synopsis is provided to DOE-BETO within one week of completing the initial and intermediate site validations. It is not produced following the final on-site validation, as there is no subsequent budget period to negotiate.

2. An initial/intermediate/final validation report that documents the results of the technical performance validation tests, as well as the process design and cost estimate 
assessments - Each of these validation reports is provided to DOE-BETO within 30 days after the completion of an on-site validation effort (or stage gate meeting as applicable). By definition, the on-site validation effort is completed when the validation team receives all the data and completed analysis from the project team, which is not necessarily the last day of the on-site visit.

Technical validation topics included in the validation report include results from the demonstrated experimental work, observed technical variances, analytical techniques, standard operating procedures, and project team capabilities. Techno-economic and lifecycle analysis topics included in the validation report include potential product market opportunities, selection of envisioned commercial-scale plant size, process overview, mass and energy balances, capital and operating costs, minimum product selling price calculations, and greenhouse gas emissions and potential reductions calculated via established lifecycle analysis methodologies.

The TechFin tables for the project are also included in the validation report as a point of reference and to make the validation report a standalone document that includes all information relevant to the on-site validation proceedings. If updates are made to the TechFin table as part of the validation process, the updated information is incorporated as new rows or columns in the table, and all the original table data are retained to provide consistency and transparency.

\section{Validation Protocols}

\subsection{Protocol for Proposing Changes (Change Control Protocol)}

The goal of the FOA should be reflected in the work demonstrated during the validation process. The validation process is intended to assess technical performance and cost improvements within the context of the FOA goals, as defined in the negotiated award. It is understood that research on such processes is ongoing and that consequently some changes to the overall integrated process that do not directly relate to the larger FOA goal itself may occur over the course of the project. In such cases, the recipient must provide DOE-BETO and the validation team with adequate information about the modified process, including any proposed changes to cost and performance assumptions, and the basis for these, in order to enable the validation of the key performance and cost metrics. The recipient must also demonstrate that any improvements based on sources other than those expressly included within the approved project scope were not paid for with DOEBETO or project cost-share funds.

Change control protocols must be followed before implementing any changes in the feedstock, process, cost, or project plan during the project. The recipient needs to describe any proposed changes to the feedstock, process, or project plan relative to what was described in the application, and the recipient must submit this information in the form of a change request to DOE-BETO before adoption for a determination as to the level of change control that must be followed. Any changes to the process - including equipment, materials used, and cost estimates - must be described in quarterly reports or by other written means no later than three to four weeks before the next on-site validation team visit.

As appropriate, revised performance metrics may also be proposed and the basis and assumptions for each revised metric must be described. Changes to project scope, technical targets/tables, schedule, or budget that impact the award may need to be approved by the DOE- 
BETO contracting officer. Changes that do not rise to that level need to be approved by the DOE-BETO project officer, and they may require changes to the project's project management plan. DOE-BETO informs both the validation team and the recipient when the changes have been accepted.

If DOE-BETO approves the changes, the recipient is required to submit revised TechFin tables with revised benchmark and/or target data that represent the updates (as well as any appropriate supporting documentation) along with a detailed description of how the process changes differ from what was specified in the original application or negotiated award.

Any changes to the project targets, research plan, methods, or the statement of project objectives must have prior DOE-BETO approval and must be described in detail in quarterly reports or by other written means at least three to four weeks before scheduling the next on-site validation visit.

\subsection{Protocol for Initial Validation}

DOE and the validation team contact the recipient within 10 business days of the official start date of the project, which is the day the award is signed by the DOE-BETO contracting officer.

The initial on-site validation is performed to (1) validate technical benchmark data, (2) replicate the benchmark performance data before improvements funded through the project, and (3) (after the benchmark process performance has been confirmed or re-established) replicate the benchmark process details and techno-economic information, with the recipient using the project's approved techno-economic calculation methods.

At the initial validation, feedstock material (raw and/or pretreated) for use throughout the validation process needs to be segregated as a lot and labeled, and adequate tracking must be maintained so that the validation team can verify that the same feedstock material is being used during each phase of the validation process. The validation team reviews the protocol for feedstock tracking during the first site visit. If new lots of feedstock material (raw or pretreated) are obtained or made during the project, their composition (and, if pretreated, the conditions under which they were produced) must be provided to the validation team in the quarterly reports or by other written means before scheduling the next on-site validation visit. Any materials made via a new process must be analyzed and controlled as per the feedstock control methodology described above.

\subsubsection{Technical Performance Validation}

The recipient is required to perform the full proposed process operations described in the application and/or negotiated in the award. A complete analysis is used to determine the performance of the benchmark process or processes. It is expected that new (validated) benchmark data should be able to replicate the data presented in the application (or agreed to during award negotiations) to within a 5\% coefficient of variation. Improvements to process economics made during the project are measured against the benchmark performance verified during the initial validation phase.

Key aspects of this part of the validation procedure include: 
1. The recipient, validation, and DOE-BETO teams schedule an on-site visit or visits by the validation team to observe ALL experiments.

2. Validation experiments for each process must occur at least in duplicate and preferably in triplicate (if equipment and available resources allow).

3. Compositional analysis of relevant process streams must be provided in sufficient detail to demonstrate the mass balance.

4. Analytical procedures for determining the composition of all necessary streams (or for analyzing time point samples obtained from a batch process) must be provided.

5. A stock (or stocks) of the feedstock, enzyme preparation(s) (or enzyme production strain), and fermentation strain(s) or catalyst(s) used in the initial benchmarking validation experiments must be maintained (as appropriate) to enable direct comparisons to be made with the improved process or processes to be developed and evaluated during the intermediate validation and final validation phases.

6. A summary of experimental results, including data tables and product conversion yields is sent to the validation team once data analysis is complete.

7. If technical performance or replication of application data cannot be verified during the on-site visit, the recipient, DOE-BETO, and validation team may agree to a period of time to develop the data in question, followed by a second review by the validation team.

\subsubsection{Process Design and Cost Validation}

The primary purpose of the initial techno-economic validation is to verify the benchmark process data and the techno-economic assumptions and methodology, including process details and cost estimates related to the overall process and individual process steps.

Key aspects of this part of the validation procedure include:

1. The recipient provides the technical and economic tables showing the (1) benchmark, intermediate, and final targets and (2) supporting information based on the results of the benchmark technical validation experiments. Supporting information should include, at a minimum, the mass and energy balance data, process flow diagrams, and costing information used to complete the TechFin tables provided in the application.

2. The project and validation teams review the TechFin tables along with supporting documentation for the benchmark process. The validation team determines whether any additional information is needed to fully understand the benchmark or to perform subsequent validations (e.g., lack of or insufficient details regarding material balances, process flow diagrams, or costs).

3. If process details and cost estimates cannot be verified because of insufficient information, the recipient, DOE-BETO, and validation team may agree to a period of time to develop the missing information, followed by a second review by the validation team.

The validation team determines whether any information required for fully understanding and verifying the proposed improvements is missing or lacking. If experimental data, process details, or cost estimates cannot be verified because incomplete information, the recipient, DOE-BETO, 
and validation team may agree to a period of time to develop the missing information to enable a second review by the validation team. The rescheduled validation is typically scheduled to occur within three to four weeks after the date of the first initial validation. Ultimately, a file of the documentation used to validate the project's process improvement progress is created and stored at DOE's Golden Field Office. In the event of an unsuccessful initial validation, refer to the protocol section (Section 5.3) for dealing with unsuccessful validations.

\subsection{Protocol for Intermediate Validation}

The intermediate validation is scheduled approximately $12-18$ months after the project is initiated but before the go/no-go or stage gate decision point as determined by the project schedule. This second on-site validation is performed by the validation team to document (1) improvements to the process or processes and (2) improvements in outside process or processes that impact the performance of the project's applicable process steps, and (3) progress of the project as measured by intermediate targets in the TechFin tables. The process design and cost validation takes place in tandem with the technical performance validation experiments, and it incorporates the demonstrated experimental data-by applying the project's cost estimation methodology — to determine the level of process cost reduction achieved.

Any changes to the process or process steps developed since the initial on-site validation must be highlighted in communications to the validation team before the intermediate site visit. In such cases, appropriate additional supporting documentation may need to be provided to the validation team. Note that any changes should already have been reported in quarterly reports and approved by DOE-BETO through the change control process described in the Section 5.1 of this document. Any changes to the process outside of the project scope are reviewed at this time, along with any new supporting documentation.

\subsubsection{Technical Performance Validation}

The methodology is identical to that employed for the initial validation, as described above, including the key aspects of the validation procedure spanning scheduling of an on-site visit by the validation team through obtaining a detailed summary of experimental data and data work-up into target performance metrics.

\subsubsection{Process Design and Cost Validation}

Again, the approach follows that described for the initial validation, with the validation team reviewing cost estimates obtained during the intermediate validation against both the original (benchmark) project target and the intermediate project target. For this phase of the validation, the recipient updates the technical and economic tables, detailing the current (intermediate) results based on the actual process improvements. The recipient also provides appropriate supporting information (e.g., updated energy and material balances, sizing calculations, and performance calculations) sufficient to enable the validation team to validate the accuracy of the technical and economic tables. The project and validation teams review the TechFin tables along with any supporting documentation on the improved process.

The validation team again determines whether any information required for fully understanding and verifying the proposed improvements is missing or lacking. If experimental data, process details, or cost estimates cannot be verified because of incomplete information, the recipient, 
DOE-BETO, and validation team may agree to a period of time to develop the missing information to enable a second review by the validation team. The validation is typically rescheduled to occur within three to four weeks after the date of the first intermediate validation. Ultimately, a file of the documentation used to validate the project's process improvement progress is created and stored at DOE's Golden Field Office. In the event of an unsuccessful initial validation, please refer to the protocol section (Section 5.4) for dealing with unsuccessful validations.

\subsection{Protocol for Final Validation}

The third and final on-site validation is conducted within two months of the completion date of the award (before or after, as is feasible), following procedures that are similar to those used in the previous phases of the project validation. The purpose of this final validation is to assess the final performance of the process improvements addressed within the scope of the award. This validation also documents improvements in processes impacting the performance and cost attributes of the overall improved system. Results from these final validation demonstrations are compared to the initial and intermediate validation results. The recipient must reserve sufficient project funds to allow this final validation and associated site visit to occur.

\subsubsection{Technical Performance Validation}

The DOE-BETO, project, and validation teams schedule an on-site visit during which validation team members observe all final process performance and validation demonstrations. The required supporting documentation (which is similar to what was provided in the previous validations) must be supplied to support this final validation, and a summary of the validation test results including data tables must be provided to the validation team after completing the experimental data analysis.

\subsubsection{Process Design and Cost Validation}

The recipient again prepares and provides to the validation team updated technical and economic tables showing the final project results in addition to the benchmark and intermediate validation data and targets. In support of these updated tables, the recipient provides an updated package of supporting information (material and energy balances, new equipment sizing calculations, new yield calculation, etc.) based on the results of the final validation demonstrations.

As before, any changes to the process or process step(s) also must be documented, and new supporting documentation provided as necessary. If process details or cost estimates cannot be verified due to a lack of information, the recipient, DOE-BETO, and validation team may allow the recipient to develop the missing information over a defined period of time to enable a second review by the validation team to occur, ideally within three to four weeks after the date of the first final validation. A file of the documentation used to validate the project's final process performance improvement progress is created and stored at DOE's Golden Field Office. In the event of an unsuccessful final validation, please refer to the protocol for dealing with unsuccessful validations outlined below.

\subsection{Protocol for Dealing with Unsuccessful Validations}

In the event that any validation demonstrations do not meet the agreed upon validation criteria during a site visit, the recipient and DOE-BETO discuss options for addressing the failed 
validation that could involve remediation steps, including a return visit by the validation team to repeat the validation demonstrations. No more than one return visit is allowed during any phase of the validation process.

Examples of validation phase failure include but are not limited to:

1. Inability to repeat results achieved in earlier experimental demonstrations

2. Unreliable data quality as the result of inadequate implementation of quality assurance and quality control procedures

3. Inability to meet the project target or targets specified in the application or agreed to during award negotiation.

The magnitude of performance variance constituting a failed validation demonstration is defined after the validation team has determined the level of variance associated with the instruments and methods used to measure performance. Typically, the return visit is scheduled within three to four weeks of the original visit. If the return visit must be delayed for more than four weeks, the schedule and the reasons for the extended time must be submitted to DOE-BETO for approval. If the project is unable to achieve all of the key performance metrics after two visits, the metrics are considered not to have been met and the validation is deemed unsuccessful.

When return visits are required to complete the technical performance and process economics elements of a specific phase of project validation, the validation report must also contain:

1. An explanation of why the return visit was required

2. The appropriate data gathered from both validations visits

3. An analysis or description of any impacts the return visit had on the project (scope, schedule, or budget)

4. Any recommendations for increased monitoring of the project or corrective actions that DOE-BETO might consider discussing with the recipient to reduce the likelihood that return validation visits are needed later on in the life of the project. 


\section{Conclusions}

Based on more than 40 project validations that have been conducted for DOE-BETO over the past decade, the following observations have been made while developing, using, and optimizing the validation process:

1. Including language in the FOA regarding the validation requirement is critical for a successful validation process and ensuring the applicant clearly understands the expectations required by this process.

2. Completing the pre-validation work before the on-site validation visits is crucial for achieving a productive on-site visit.

3. The validation process provides an assessment of project risks and opportunities to all stakeholders.

4. The validation process compiles the project-level data needed to perform analysis across the DOE-BETO project portfolio.

5. The validation process contributes significantly to informed decision making by enhancing the amount of and quality of project information available to DOE-BETO. Validations provide independent, credible, and objective analysis of project technologies to help inform these decisions.

6. The validation process ensures technical and economic information is systematically and deliberately used to understand project tradeoffs, benefits, and risks.

7. The validation process aligns project expectations and outcomes with DOE's expectations and goals.

8. Validations track project performance and progress over the life of a project. Demonstration of key technical and economic performance metrics establish the validation framework, and these metrics are used to evaluate and track the performance of the project.

9. Validations enable comparison of project performance to the state of technology benchmarks and design cases on a variety of technical, economic, and environmental dimensions.

10. Unsuccessful validations can provide the basis for DOE-BETO termination of a failing project, and an associated savings of government money.

The 2015 DOE-BETO Peer Review Panel for the conversion program unanimously supported the importance of the validation process and considers it a program strength. The panel recommended that the process continue and that the overall procedure be accurately documented (DOE 2016). This document serves as the accurate documentation of the validation process for DOE-BETO. 


\section{References}

DoD (Department of Defense). 2017. The Defense Acquisition Guidebook (DAG), Chapter 3. Accessed June 6, 2017:

https://www.dau.mil/guidebooks/Shared\%20Documents/Chapter\%203\%20Systems\%20Engineer ing.pdf.

DOE (Department of Energy). 2016. 2015 Peer Review Report: Review Panel Summary Report and Project Results. Accessed June 6, 2017:

https://energy.gov/sites/prod/files/2016/04/f30/2015_peer_review_report.pdf.

DOE-BETO (U.S. Department of Energy Bioenergy Technologies Office). 2008. Managing Design and Construction Using Systems Engineering for Use with DOE O 413.3A. Accessed June 6, 2017: https://www.directives.doe.gov/directives-documents/400-series/0413.3-EGuide01/@@images/file.

NASA (National Aeronautics and Space Administration). 2007. Systems Engineering Handbook. Accessed June 6, 2017: http://www.acq.osd.mil/se/docs/NASA-SP-2007-6105-Rev-1-Final31Dec2007.pdf. 


\title{
Appendix A: Examples of Confidentiality Agreement Documents
}

\author{
w돌 \\ COI NREL \\ Example.doc
}

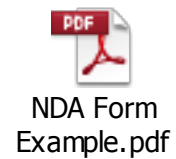




\section{Appendix B: Example of TechFin Tables Template}

X

Sample TechFin

Tables.xlsx 


\section{Appendix C: Example of a FOA, including Language Regarding the Validation Process

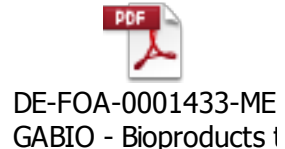




\title{
Appendix D: Example of a FOA-Specific Validation Plan
}

\author{
DOF \\ MegaBio_ValidationPI \\ an_Final.pdf
}




\section{Appendix E: Example of a Project-Specific On-Site Requirements Document \\ W슬 \\ Sample On Site \\ Validation Requiremer}

